\title{
CHOOSING COLORS FOR WORK-CENTERED SUPPORT SYSTEMS FOR COMMAND AND CONTROL USING A VISUAL SEARCH TASK
}

Gina
Thomas-Meyers
AFRL/HECS
2698 G Street
WPAFB, OH
45433-7604

\begin{tabular}{|c|c|c|}
\hline Allen & Samuel & Jeffrey \\
\hline Nagy & Kuper & Wampler \\
\hline Wright State University & AFRL/HECS & AFRL/HECS \\
\hline Department of Psychology & 2698 G Street & 2698 G Street \\
\hline 3640 Colonel Glenn Hwy. & WPAFB, OH & WPAFB, OH \\
\hline Dayton, Ohio 45435 & $45433-7604$ & $45433-7604$ \\
\hline
\end{tabular}




\begin{abstract}
The use of information technology to support work and aid in decision making has brought about new ways of displaying information on computer screens. The more information required, the more complex those displays have become. Goals of this research project were to 1) develop and test basic methodology for recommending complex display color coding and 2) to use results from that methodology to recommend specific changes to the Work-Centered Support System for Global Weather Management (WCSS-GWM) application. The on-going research shows how a simple laboratory search technique may be used to recommend chromaticities and other characteristics for use in current and future systems.
\end{abstract}

\title{
CHOOSING COLORS FOR WORK-CENTERED SUPPORT SYSTEMS FOR COMMAND AND CONTROL USING A VISUAL SEARCH TASK
}

Early display designers were constricted by availability and expense of display technology. Color displays, when first available, were cost prohibitive. As technology improved and costs decreased, display complexity increased. Design was guided by 'engineering judgment,' 'common sense,' and the way things had been done on paper. In response to user problems and needs, human factors professionals have been increasingly involved in display design. More and more, interface issues began to be addressed empirically. Human factors display guidelines have been developed (for an example, see Helander, 1987) and are now regularly applied to new technological advances.

Human factors experts have developed some general guidelines regarding the use of color in displays. In general, human factors guidelines recommend against using more 
than ten colors for the simple coding of information and that color use should be as limited as possible given the particular application (Helander, 1987). Color is particularly useful in allowing the user to easily find a particular symbol in a dense display, for drawing attention to particular information, and for allowing the user to rapidly sort and/or categorize information. Guidelines also suggest that red, green, and yellow be reserved for representing danger, safe conditions, and caution, respectively (Helander, 1987). Current military standards (DoD, 1989) specify that "red shall be used to alert an operator that the system or any portion of the system is inoperative or that a successful mission is not possible until appropriate corrective or override action is taken." Emergency conditions that require immediate action are to be indicated by flashing red. Yellow is to be used to indicate conditions that are "marginal" or to "alert the operator to situations where caution, recheck, or unexpected delay is necessary." Satisfactory conditions are to be indicated with green. White is reserved for indicating "system conditions that do not have 'right' or 'wrong' implications." Blue is approved for use as an "advisory light, but preferential use of blue should be avoided." Color coding may also be used to "differentiate between classes of information in complex, dense, or critical displays [but] ... shall not conflict with the [above] color associations" (DoD, 1989). Human factors experts also recommend that color coding only be used for identification of symbols with spatial frequencies greater than about two cycles per degree of visual angle (particularly saturated blue) and that red and green not be used in the periphery of a display (Helander, 1987).

More recently, human factors guidelines (Bennett, Nagy, and Flach, 1997) have extended into methods of adapting to new technology in displaying information. Human 
factors experts began to realize that the basic guidelines for display were sufficient for displaying data (such as system states) but not necessarily for displaying information (such as combinations of data needed for planning action). For complex or detailed displays, human factors experts recommend layering the information with respect to importance. In other words, the most important information should be the most prominent visually. Methods of display (i.e. length comparisons vs. volumetric comparisons) which provide the most effective means of quantifying data are fairly well understood. Attention-based methods of design focus on the graphical representations in a display and aspects related to separability, integrability, and configurality of displayed information. Human factors experts have agreed that perceptual proximity and processing proximity should be compatible. In addition, the notion that the choice of which information to display must depend on the interaction between the domain and the interface is prominent in the human factors literature (Bennett, Nagy, and Flach, 1997).

The use of information technology (IT) has grown exponentially, both in business and in government. This technology provides support to workers in terms of automating duties, expediting information retrieval, allowing ease of making changes to existing forms and documents, making fast and easy calculations, allowing tracking of work, displaying the status of systems, etc. All of the information necessary to provide this support must be displayed in a limited amount of space.

One such system currently being applied as a demonstration project in a command and control domain is the Work-Centered Support System Global Weather Management (WCSS-GWM) application that was developed under the Air Force Research Laboratory Human Effectiveness directorate's program called Global Air Mobility Advanced 
Technology (GAMAT). This technology demonstration is an example of a WorkCentered Support System (WCSS; Eggleston and Whitaker, 2002; Young, et. al, 2000). WCSS technology is a new cognitive-science-based analysis and design methodology for developing human-computer interfaces that enable very high user productivity, especially in dynamic and information-dense environments such as command and control. The WCSS technology leverages cognitive work analysis and advanced user interface design techniques to provide intuitive user interfaces customized based on the work requirements. The WCSS approach is designed to develop human-computer interfaces that support rapid user adaptation to both routine and non-routine/unexpected events by providing relevant context and intuitive and directly manipulable affordances for action.

The Work-Centered Support System for Global Weather Management (WCSSGWM) is a software client application developed with a goal of minimizing the impact of changing weather conditions on planned and on-going missions (see Fig. 1). The WCSSGWM software allows users to select and inspect missions of interest and provides intelligent-agent-based alerts when weather phenomena may impact those missions. It provides a consolidated view of weather and air mission data. It provides the user with the ability to select from a variety of weather and flight related data and arrange them as different layers on a map of the world in flexible ways to efficiently support dynamic work requirements. WCSS-GWM has been demonstrated to be useful for weather forecasters to select relevant areas for prediction, for weather support personnel to understand which weather changes might affect current and planned flights, and for flight managers to know when a flight plan might need to be adjusted due to current or impending weather conditions (see Scott, et. al, in publication). 


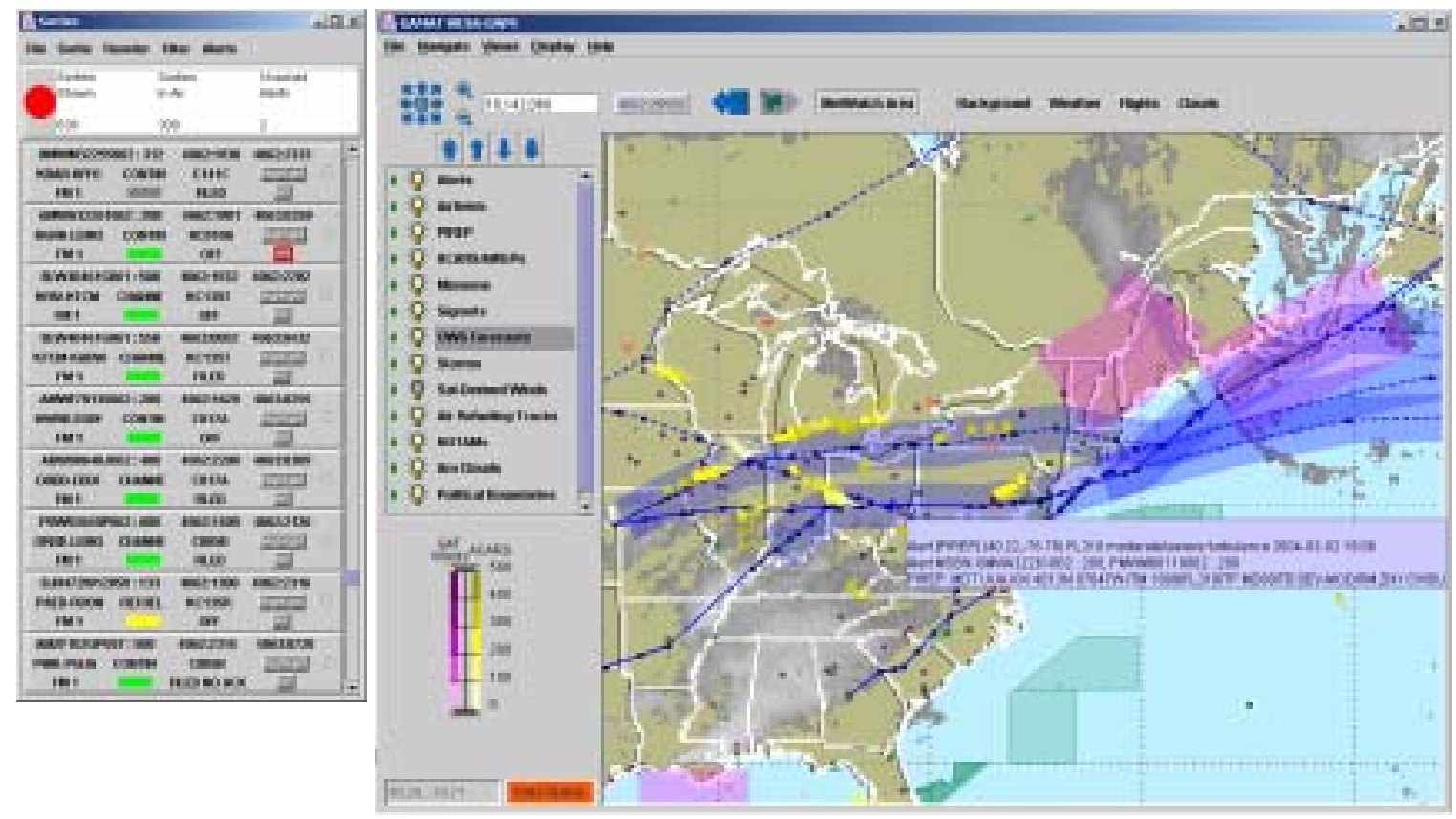

Figure 1 - Screen shot of the Work-Centered Support System for Global Weather Management

The WCSS-GWM application is being expanded for use by other command and control users in both the local domain and external domains. The display is already quite complex in its use of color for the coding of layers and alerting information. Subsequent iterations of this application are expected to require even more types of information to be displayed.

Although the work-centered concept is a particularly useful method of displaying relevant information for use in decision support, for any system, when graphical information is coded by color, symbology or geometry, "no matter how intelligent the choice of information, no matter how ingenious the encoding ... the graph is a failure if the visual decoding fails" (Cleveland, 1985). It is, therefore, quite important to include issues of visual perception in the development of any new display.

When several types of information are displayed, the user must visually search for the information that is most relevant to the task at hand. In some cases, the relevant item is quite easy to find; it seems to stand out from other elements of the display. In other 
instances, search is more perceptually laborious. In past literature, easy searches have been referred to as parallel and more difficult searches have been referred to as serial (Treisman, 1998; Treisman, 1988; Treisman, 1991; Northdurft, 1993; Hulleman, et al. 2000; Treisman and Gelade, 1980; Quinlan, 2003; Treisman and Souther, 1985). These descriptions imply that there is something inherently different about the two types of stimuli. Although vision researchers are not in agreement that these differences in search difficulty are truly due to switches between parallel and serial processing, there is wide agreement that the differences exist and that there is something about the types of stimuli and combinations of stimuli that lead to them.

In general, when information is displayed, there are three levels of search criteria. The higher levels cannot be achieved unless all lower level criteria are met. At the lowest level, a stimulus must be visible. For example, someone looking at a radar display of various aircraft must be able to see the blips on the screen. At the next level, a stimulus must be discriminable. For example, if a display used shape to indicate the types of aircraft present, it would be important for the user to be able to detect those shape differences if decisions were to be made based on aircraft type. At the highest level, a stimulus must be conspicuous. For example, if it is important that a warning signal be noticed, that signal must be displayed in such a manner that, if it appears, it will stand out from other elements of the display. In the WCSS-GWM display, the alert is such a signal.

There are a number of objectives of the current research. One specific goal is to demonstrate that a basic research methodology such as a search task can be useful for designing and evaluating coding in an applied setting. In this particular case, the goal is 
to use a search task to test the effects of the use of color and transparency in a display.

The research effort to date consists primarily of the development of a search task to test the effects of color and transparency combinations in the WCSS-GWM display.

\section{METHOD}

To date, pilot data only have been collected for this research program. The following methods were employed in the collection of those data.

\section{Participant}

The author (female) acted as subject in the collection of pilot data.

\section{Apparatus}

Stimuli were generated using a JAVA computer program written specifically for use in this research and displayed on flat panel personal computer monitor similar to those used by the users of the WCSS-GWM display. The pilot studies were run in a typical office setting.

\section{Procedure}

Stimuli (target and distractors) were presented on one of two background types. The first background type (referred to as original) was generated from screen shots of the WCSS-GWM display. Initially, interviews of current and potential users of the WCSSGWM weather tool were conducted to assure that any recommended color changes to the display would not violate user expectations. The second background type (referred to as recommended) was like the original background except that colors were de-saturated. This choice was deemed to be the simplest change that could be made that reflected past research findings. The two background types were present at all times during the experimental block and were displayed with three levels of clutter - low, moderate, and 
high - generated by varying the number of layers displayed in the WCSS-GWM screen shots.

The target consisted of a red circle similar to the alert in the WCSS-GWM display and the distractors consisted of a variety of shapes (other that circles) similar to some of the symbology used in WCSS-GWM. In addition to varying the level of background clutter, stimuli were presented in set sizes (target and distractors) of $3,6,18$, or 54. The colors of the distractors in one condition were similar to the current WCSS-GWM symbol colors and, in another condition, selected based on the interviews and a brief review of current human factors literature.

In a typical trial, a fixation cross was presented at the center of the display background, and the subject focused on that cross. The target and distractor(s) appeared, and the subject pressed enter when she located the target. The target and distractor set then disappeared, and the subject placed the cursor in the quadrant where she believed that the target had been. Inaccurate trials were repeated; only accurate trials were included in the analysis; less than $10 \%$ of trials were inaccurate in all conditions. Data from inaccurate trials were, however, saved so that they would be available for post hoc analyses, if needed.

Eight blocks of sixty trials were completed representing two blocks of each combination of set size (3, 6, 18 and 54) and background color (original and recommended). Each blocks consisted of ten trials of each combination of distractor color (original and recommended) and level of clutter (low, moderate, and high). 


\section{RESULTS}

There was a significant main effect of de-saturating the background color for all set sizes (see figure 2) and across all levels of clutter (see figure 3), $f=24.6, p<0.05$. There were also significant main effects of both set size, $\mathrm{f}=5.9, \mathrm{p}<0.05$, and background clutter, $f=7.1, p<0.05$, as would be expected. Although figure 3 shows a hint of an interaction between background color and clutter, this interaction was not statistically significant, $f=2.4, p>0.05$.

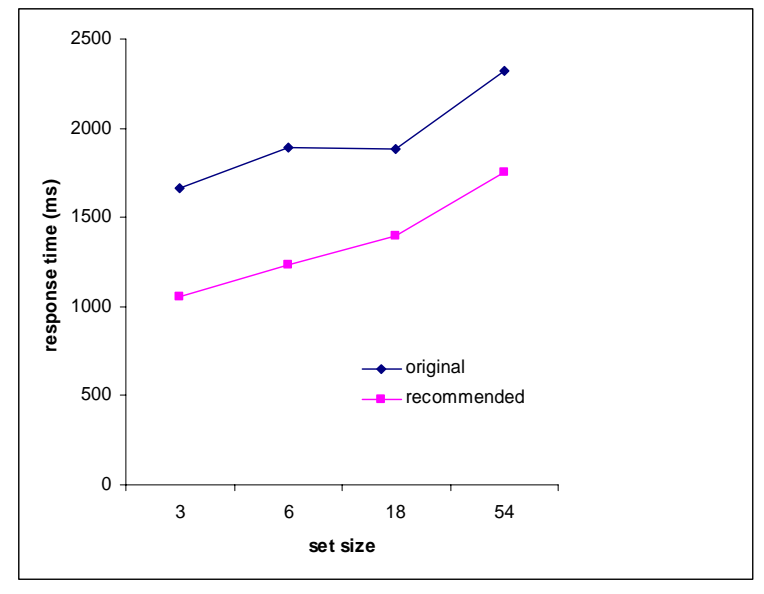

Figure 2 - Mean response times by set size averaged across all three levels of background clutter for original and recommended (de-saturated) background colors

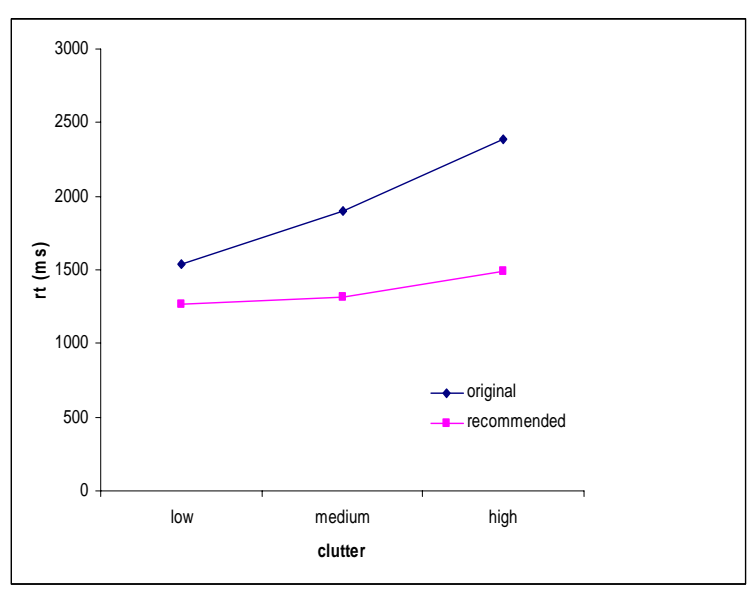

Figure 3 - Mean response times by clutter averaged across set size for original and recommended (de-saturated) background colors

There were no significant main effects for symbol (distractor) color, $f=0.3, p>$ 0.05. The only set size for which there was any benefit of the new distractor colors was the largest set size, and that benefit was not statistically significant, $f=1.2, p>0.05$. 


\section{DISCUSSION}

The purposes of this pilot research were to verify that it is reasonable to use a basic laboratory research paradigm in order to make specific recommendations to a very complex and information-rich display and to determine color sets that might improve the efficiency of such a display so that those sets may be further tested.

It seems that the search task used in the pilot research is an effective way to determine which display modifications are potentially beneficial. In addition to providing evidence that the de-saturation of the display background is likely to have a positive effect on the ability of the user to detect an alert, the search task also allowed us to pre-test a set of symbol colors in a laboratory situation before applying them to an actual display. The validity of the search results was supported by the fact that the task was sensitive to the expected effects of clutter and set size. This task is currently being applied in further experiments in which effects of color, background, clutter and transparency are all being investigated. 


\section{REFERENCE}

Bennett, K. B., Nagy, A. L., and Flach, J. M. (1997). Visual Displays. In Handbook of Human Factors, $2^{\text {nd }}$ ed. G. Salvendy (ed). New York: John Wiley and Sons.

Cleveland, W. S. (1985). The Elements of Graphing Data. Belmont, CA: Wadsworth. Department of Defense (1989). Military Standard: Human Engineering Design Criteria for Military Systems, Equipment, and Facilities. MIL-STD-1472D.

Eggleston, R. and Whitaker, R. (2002). Work Centered Support System Design: Using Frames to Reduce Work Complexity. Proceedings of the Human Factors and Ergonomic Society $46^{\text {th }}$ Annual Meeting.

Helander, M. G. (1987). Design of Visual Displays. In Handbook of Human Factors, G. Salvendy (ed). New York: John Wiley and Sons.

Hulleman, J., teWinkel, W, and Boselie, F. (2000). Concavities as basic features in visual search: Evidence from search asymmetries. Perception and Psychophysics, 62-1, 162-174.

Nothdurft, H. C. (1993). Saliency effects across dimensions in visual search. Vision Research, 33, 839-844.

Quinlan, P. (2003). Visual Feature Integration Theory: Past, Present, and Future. Psychological Bulletin, 129(5), 643-673.

Scott, R., Roth, E. M., Deutsch, S. E., Malchiodi, E., Kazmierczak, T., Eggleston, R.G. and Kuper, S. R., Whitaker, R. (in publication). Work-Centered Support Systems: A Human-Centered Approach to Intelligent System Design. IEEE Intelligent Systems. 
Treisman, A. (1988). Features and Objects: The Fourteenth Bartlett Memorial Lecture. The Quarterly Journal of Experimental Psychology, 40(2A), 201-237.

Treisman, A. (1998). The perception of features and objects. In Visual Attention, Wright, R. D., ed. Oxford University Press: London, 26-54.

Triesman, A. (1991). Search, Similarity. And Integration of Fetures Between and Within Dimensions. Journal of Experimental Psychology: Human Perception and Performance, 17-3. 652-676.

Treisman, A. and Gelade, G. (1980). A Feature-Integration Theory of Attention. Cognitive Psychology, 12, 97-136.

Treisman, A. and Souther, J. (1985). Search Asymmetry: A Diagnostic for Preattentive Processing of Separable Features. Journal of Experimental Psychology: General, $114-3,285-310$.

Young, M.J., Eggleston, R. G., and Whitaker, R. D. (2000, April). Direct manipulation interface techniques for interaction with software agents. Paper presented at the NATO/RTO Symposium on Usability of Information in Battle Management Operations, Oslo, Norway. 